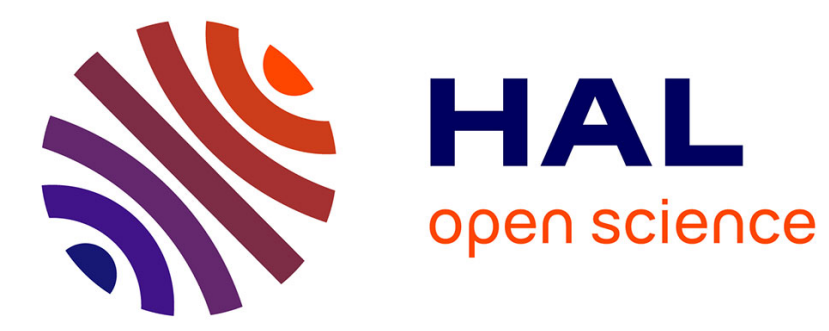

\title{
Brillouin light-scattering from poly(acrylic acid) hydrogels
}

\author{
S. Ng, L. Gan, Y. Li, T. Chieng
}

\section{To cite this version:}

S. Ng, L. Gan, Y. Li, T. Chieng. Brillouin light-scattering from poly(acrylic acid) hydrogels. Journal de Physique II, 1994, 4 (4), pp.715-722. 10.1051/jp2:1994158 . jpa-00247994

\section{HAL Id: jpa-00247994 https://hal.science/jpa-00247994}

Submitted on 1 Jan 1994

HAL is a multi-disciplinary open access archive for the deposit and dissemination of scientific research documents, whether they are published or not. The documents may come from teaching and research institutions in France or abroad, or from public or private research centers.
L'archive ouverte pluridisciplinaire HAL, est destinée au dépôt et à la diffusion de documents scientifiques de niveau recherche, publiés ou non, émanant des établissements d'enseignement et de recherche français ou étrangers, des laboratoires publics ou privés. 
Classification

Physics Abstracts

$62.90-78.35-82.70$

\title{
Brillouin light-scattering from poly(acrylic acid) hydrogels
}

\author{
S.C. Ng, L.M. Gan $\left({ }^{*}\right)$, Y. Li and T.H. Chieng $\left({ }^{*}\right)$ \\ Department of Physics, National University of Singapore, Kent Ridge, Singapore 0511
}

(Received 13 July 1993, received in final form 22 December 1993, accepted 3 January 1994)

\begin{abstract}
The Brillouin shift and width for poly(acrylic acid) hydrogels have been obtained as a function of gel network volume fraction using a five-pass Fabry-Pérot interferometer. A conspicuous peak has been observed in the Brillouin shift at high volume fraction. The results are discussed in the light of a theory for the Brillouin light-scattering from gels proposed by Marqusee and Deutch.
\end{abstract}

\section{Introduction.}

In a recent study on Brillouin light-scattering from polymer gels [1], our previous measurements of the dependence of the true Brillouin shift $\nu_{\mathrm{B}}$ and width $\Gamma_{\mathrm{B}}$ on the volume fraction $\phi$ of the gel network for poly(vinyl alcohol)(PVA) [2] and poly(vinyl chloride)(PVC) [3] gels were compared with the theory of Marqusee and Deutch(MD) [4], incorporated with a new assumption that the bulk modulus of the gels scales as $\phi$. It has been found that the measurements of $\nu_{\mathrm{B}}$ and $\Gamma_{B}$, over most of the range of $\phi$ are amazingly consistent with the essential features of the predictions of the MD theory for the case of weak coupling and strong frictional damping [1]. However, the weak coupling case is an abbreviated version of the theory and therefore the experimental data of PVA and PVC gels do not constitute a complete test for the MD theory. There are other features which occur in the case of strong coupling but not in weak coupling. For instance, the MD theory for the case of strong coupling and frictional damping predicts the presence of a peak in $\nu_{\mathrm{B}}$ at high $\phi$, a feature which is missing in the weak coupling case. In order to explore this more intricate possibility we undertook a study on another gel system, poly(acrylic acid) (PAA).

$\left.{ }^{*}\right)$ Department of Chemistry, National University of Singapore. 


\section{Experimental.}

The materials used in this work, acrylic acid(AA) and dibenzyl ketone(DBK), were obtained from Tokyo Chemistry Industry. AA of purity higher than $99 \%$ was distilled under reduced pressure before use. DBK of purity higher than $98 \%$ was used as a photoinitiator without further purification. Samples of the PAA hydrogel were prepared in the following manner. Solutions of known masses of AA, doubly distilled water and DBK $(0.3 \%$ of the mass of AA) were introduced into clean glass tubes and purged with purified nitrogen gas for about 10 minutes before being sealed. The sealed samples were then polymerized by ultra-violet light. The conversion of polymerization was determined to be higher than $97 \%$. Assuming $100 \%$ conversion, the gel network volume fraction $\phi$ of the resulting PAA hydrogels can be determined quite accurately by the equation

$$
\phi=\frac{m_{\mathrm{n}} \rho_{\mathrm{f}}}{m_{\mathrm{n}} \rho_{\mathrm{f}}+m_{\mathrm{f}} \rho_{\mathrm{n}}}
$$

where $\rho_{\mathrm{n}}$ and $\rho_{\mathrm{f}}$ are the densities and $m_{\mathrm{n}}$ and $m_{\mathrm{f}}$ the masses of PAA and water respectively.

The PAA hydrogels were transparent. Those with $\phi$ less than 0.25 did not exhibit sufficient strength to be self-supporting. However, they became rubbery and glassy at higher $\phi$ values. Many studies have been made on the preparation and characterization of cross-linked PAA hydrogels $[5,6]$. It has been reported that cross-linked PAA can be separated from the uncrosslinked PAA(atactic and stereoregular forms of PAA) by extraction with dioxane followed by $20 \%$ water in dioxane [7]. Based on this method, we have found that the hydrogels with $\phi>0.25$ could not be solubilized in the solvents but formed highly swollen gels. These swollen PAA gels were further disintegrated into small fragments in hot $1 \%$ Triton $\mathrm{X}-100$ (non-ionic surfactant) aqueous solution. This indicates that the PAA hydrogels may have cross-linked network. The gel network may be formed by strong interactions between PAA chains through hydrogen bonding via carboxyl groups. In addition, the formation of some chemical (covalent) cross-links may also be possible. However, we are unable to determine the cross-link density quantitatively.

The Brillouin spectrum at room temperature for each PAA hydrogel of different $\phi$ was obtained by exciting it using a Spectra-Physics argon-ion laser operated at single mode (linewidth $\simeq 100 \mathrm{MHz}$ ) at a wavelength of $\lambda=514.5 \mathrm{~nm}$ and a power of $50 \mathrm{~mW}$. The light scattered through an angle $\theta=90^{\circ}$ was analyzed using a Burleigh DAS-1 five pass Fabry-Pérot interferometer operated with an optimized free spectral range of $33.8 \mathrm{GHz}$ and finesse of 42 , as described in detail elsewhere [8]. The optimized free spectral range was chosen to provide a sufficient separation of the Brillouin doublet and the strong central Rayleigh peak at low $\phi$ and also to prevent the overlapping of the Brillouin peaks at high $\phi$. Any measured Brillouin spectrum is the result of an unavoidable convolution between the true spectrum and the instrumental response. The effects of the instrumental resolution were removed and the true $\nu_{\mathrm{B}}$ and $\Gamma_{B}$ were obtained using an iterative convolution and least-squares fitting procedure, assuming a Lorentzian line shape for the true spectrum [9].

Marqusee and Deutch give an analytic expression for the light-scattering spectrum for polymer gels [4]. It describes two Brillouin peaks and a central diffusive peak, which are all of Lorentzian line shapes. However, the non-Lorentzian contributions to the light spectrum are not included in their expression, as they are expected to be small [4]. Figure 1 depicts the method by which three Rayleigh-Brillouin spectra for PAA hydrogel of different $\phi(0.06,0.65$ and 1.0) were computer analysed. The measured Brillouin peaks, shown as full circles on the right-hand side of the figure, were least-squares fitted with a profile given by convolving a Lorentzian line shape, representing the true Brillouin spectrum, with the measured instrumental resolution, which was obtained from the Rayleigh peak, as shown on the left-hand side of 


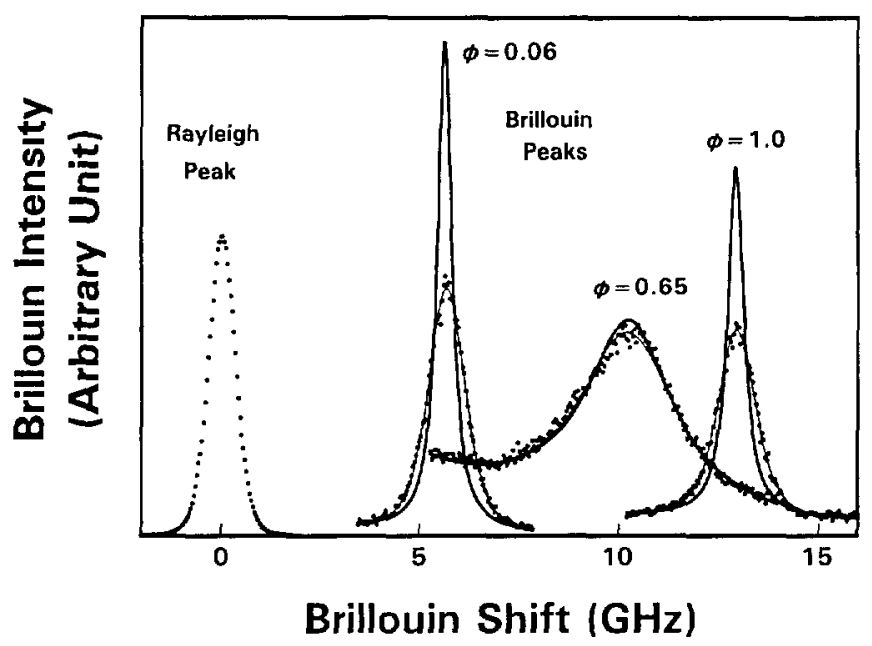

Fig. 1. - The Rayleigh-Brillouin spectra for PAA hydrogels of $\phi=0.06,0.65$ and 1.0 . The figure is fully explained the text.

the figure. A sloping linear background is also included in the fit to describe approximately any weak non-Lorentzian and diffusive contributions. The parameters of the Lorentzian and the background are optimized to obtain the best fit to the experimental data. The fitted profiles are shown in the figure as fine lines passing through the experimental points and the resulting deconvolved true spectrum as bold lines. The fitted true Brillouin shift $\nu_{\mathrm{B}}$ is equal to the resonance peak frequency of the optimized Lorentzian profile and the fitted true Brillouin width $\Gamma_{B}$ is equal to its full width at half the maximum intensity(FWHM). Note that, while the measured and true spectra have essentially the same peak frequency, their FWHMs differ markedly for narrow spectra due to the broadening effect of the instruments.

The PAA hydrogels with $\phi$ less than 0.56 are rubberlike and their refractive indices at $\lambda=$ $514.5 \mathrm{~nm}$ were measured at room temperature using an Abbé refractometer.

\section{Results.}

The results for the Brillouin shift $\nu_{\mathrm{B}}$ at room temperature for the PAA hydrogels are displayed in figure 2. For increasing $\phi, \nu_{\mathrm{B}}$ increases slowly at first, then at $\phi \sim 0.4$ it increases more rapidly reaching a maximum near $\phi \sim 0.9$ thereafter dropping slightly as $\phi$ approaches 1 . This is in contrast to our previous results on PVA and PVC gels [2,3], as well as those of Brown et al. on solutions of poly(methylmethacrylate)(PMMA) in toluene [10], in which $\nu_{\mathrm{B}}$ increases monotonously with $\phi$.

The measurements of the Brillouin width $\Gamma_{\mathrm{B}}$ are shown in figure $3 . \Gamma_{\mathrm{B}}$ increases rapidly with increasing $\phi$ reaching a maximum near $\phi \sim 0.65$ thereafter dropping rapidly as $\phi$ approaches 1. This behaviour of $\Gamma_{\mathbf{B}}$ is similar to those of PVA, PVC and PMMA gels $[2,3,10]$.

Figure 4 shows the dependence of the measured refractive index $n$ on $\phi$. The value for PAA $(\phi=1)$ is taken from Polymer Handbook [11]. These results show that $n$ depends approximately linearly on $\phi$ and the measurements for $n(\phi)$ were parametrized in accordance with $n(\phi)=$ $n_{0}+n_{1} \phi$. The values obtained for $n_{0}$ and $n_{1}$ are given in table I. 


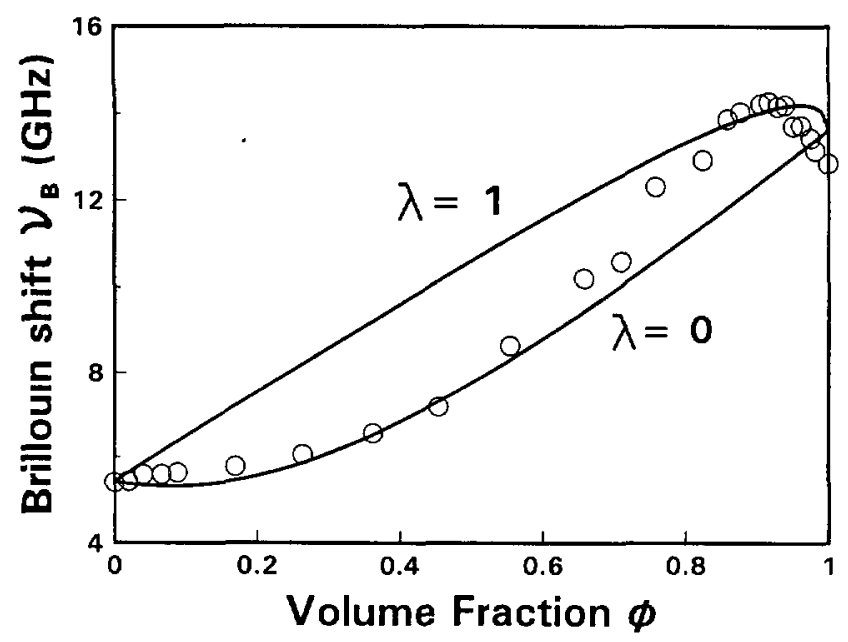

Fig. 2. - The Brillouin shift in PAA hydrogels as a function of volume fraction. Open circles are experimental data. Lines are theoretical curves for $\lambda \simeq 0$ and $\lambda=1$ (see text).

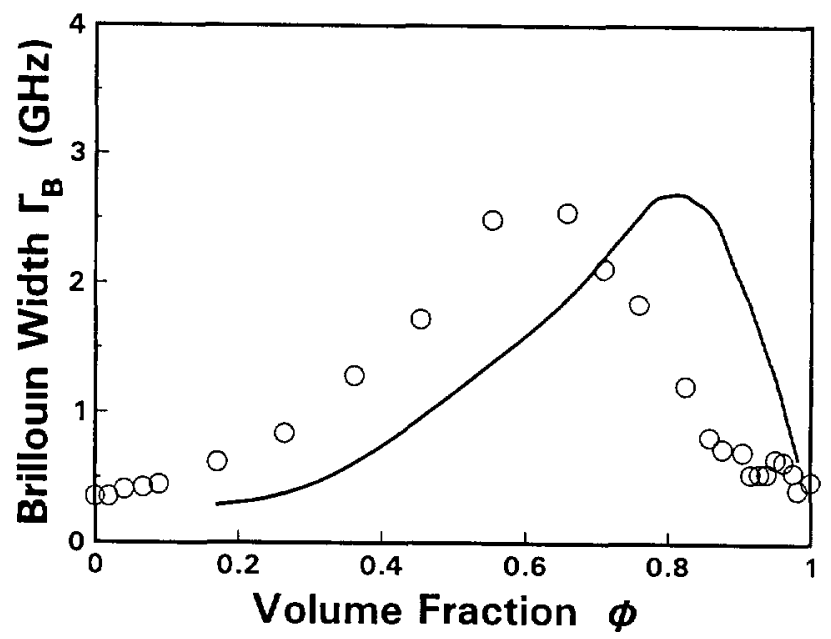

Fig. 3. - The Brillouin width in PAA hydrogels as a function of volume fraction. Open circles are experimental data. The line is a theoretical curve (see text).

\section{Comparison with theory.}

Marqusee and Deutch consider a polymer gel as a coupled polymer network-fluid system. The degree of coupling between elastic waves in the network and fluid is measured by a parameter $0 \leq \lambda \leq 1$. Two limiting cases are examined by them. The first case occurs for small frictional damping $f$ and predicts a two-mode type of behaviour for the Brillouin spectrum. In this case two pairs of Brillouin peaks should be observable, which for weak coupling $\lambda \simeq 0$, are close to the Brillouin angular frequencies $\pm \omega_{0}$ and $\pm \omega_{\mathrm{n}}$. Here $\omega_{0}=C_{0} k$ and $\omega_{\mathrm{n}}=C_{\mathrm{n}} k, C_{0}$ and $C_{\mathrm{n}}$ are respectively the speed of sound in the fluid and network and $k$ is the sound wave vector. The other limiting case is for strong frictional damping. It corresponds to a one-mode type 
Table I. - The bulk physical parameters of PAA hydrogel at room temperature. All the symbols used are defined in the text.

\begin{tabular}{||l|c|c||}
\hline \hline Parameter & Value & Reference \\
\hline$\rho_{\mathrm{f}}$ & $998 \mathrm{kgm}^{-3}$ & {$[18]$} \\
\hline$\rho_{\mathrm{n}}$ & $1212 \mathrm{kgm}^{-3}$ & {$[11]$} \\
\hline$C_{0}=\bar{C}(\phi=0)$ & $1475.5 \mathrm{~ms}^{-1}$ & {$[19]$} \\
\hline$C_{\mathrm{n}}=\bar{C}(\phi=1)$ & $3250 \mathrm{~ms}^{-1}$ & This work \\
\hline$\frac{4}{3} \eta_{\mathrm{S}}+\eta_{\mathrm{B}}$ & $2.92 \times 10^{-3} \mathrm{kgm}^{-1} \mathrm{~S}^{-1}$ & {$[19]$} \\
\hline$n_{0}$ & 1.3358 & This work \\
\hline$n_{1}$ & $1.9106 \times 10^{-1}$ & This work \\
\hline \hline
\end{tabular}

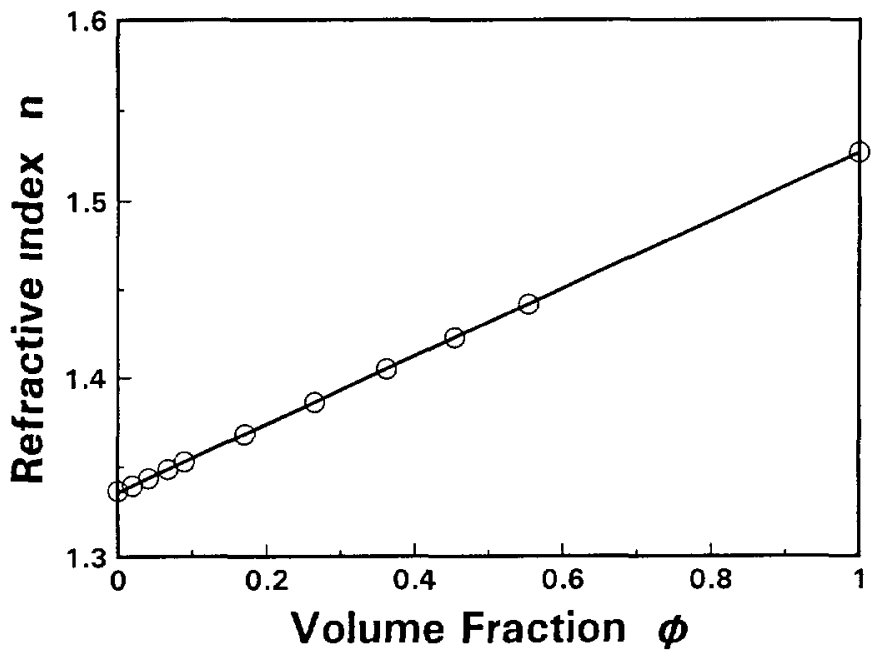

Fig. 4. - The refractive index of PAA hydrogels as a function of volume fraction. Open circles are experimental data. The line is a linear least squares fit.

of behaviour which predicts a single pair of Brillouin peaks at angular frequencies $\pm \bar{\omega}$. Here $\bar{\omega}=\bar{C} k$ and $\bar{C}$ is the average speed of sound in the gel. $\bar{C}(\phi=0)=C_{0}$ and $\bar{C}(\phi=1)=C_{\mathrm{n}}$.

The present measurements of Brillouin spectra of PAA hydrogel, like all previous measurements on PVA, PVC and PMMA gels $[2,3,10]$, show the presence of only a single pair of modes. It therefore seems appropriate to compare the results with the theory for the limit of strong friction. Marqusee and Deutch give two equations for the $\phi$ - dependence of the Brillouin shift $\nu_{\mathrm{B}}$ and Brillouin width $\Gamma_{\mathrm{B}}$ (FWHM):

$\nu_{\mathrm{B}}=\bar{C} k / 2 \pi$, where

$$
\bar{C}^{2}=\frac{C_{0}^{2} \rho_{\mathrm{f}}+\phi\left(C_{\mathrm{n}}^{2} \rho_{\mathrm{n}}-C_{0}^{2} \rho_{\mathrm{f}}\right)+2 C_{0} C_{\mathrm{n}} \sqrt{\lambda \rho_{\mathrm{n}} \rho_{\mathrm{f}}\left(\phi-\phi^{2}\right)}}{\rho_{\mathrm{f}}+\phi\left(\rho_{\mathrm{n}}-\rho_{\mathrm{f}}\right)}
$$


and $\Gamma_{\mathrm{B}}=\Gamma k^{2} / \pi$, where

$$
\begin{aligned}
2 \Gamma & =\frac{4 \eta_{\mathrm{S}} / 3+\eta_{\mathrm{B}}}{\rho_{\mathrm{f}}+\phi\left(\rho_{\mathrm{n}}-\rho_{\mathrm{f}}\right)}+\frac{\rho_{\mathrm{f}}^{2} \rho_{\mathrm{n}}^{2}\left(\phi-\phi^{2}\right)^{2}}{\left(\rho_{\mathrm{f}}+\phi\left(\rho_{\mathrm{n}}-\rho_{\mathrm{f}}\right)\right)^{3}} \frac{1}{f \bar{C}^{2}} \times \\
& \times\left\{C_{0}^{2}-C_{\mathrm{n}}^{2}+C_{0} C_{\mathrm{n}}\left[\rho_{\mathrm{f}}-\phi\left(\rho_{\mathrm{n}}+\rho_{\mathrm{f}}\right)\right] \sqrt{\frac{\lambda}{\rho_{\mathrm{n}} \rho_{\mathrm{f}}\left(\phi-\phi^{2}\right)}}\right\}^{2}
\end{aligned}
$$

Here $\eta_{\mathrm{S}}$ and $\eta_{\mathrm{B}}$ are respectively the shear and bulk viscosities in the fluid. Originally $C_{\mathrm{n}}$ in equations (2) and (3) was assumed to be only weakly dependent of $\phi$. However, in comparison with the experimental data of PVA and PVC gels, it was found that the essential features of the behaviour of the Brillouin shift and width are well described by the theory over most of the range of $\phi$ if $C_{n}$ is assumed to depend on $\phi$ as $C_{n}=\bar{C}(\phi=1) \sqrt{\phi}$. [1]. This $\phi$-dependence of $C_{\mathrm{n}}$ corresponds to an assumption that the bulk modulus of the gels scale as $\phi$, which is valid for gels with a fixed degree of polymerization [12]. Analysis of the present results is also based on the same assumption. The relevant values for the physical parameters required for the calculations are listed in table I. The resulting predictions for $\nu_{\mathrm{B}}$ have been displayed in figure 2 as lines for the limiting cases of no coupling $\lambda=0$ and maximum coupling $\lambda=1$. Note that the value of $C_{\mathrm{n}}$ has been chosen so that most of the data points fall within these two limiting curves as required by the condition $0 \leq \lambda \leq 1$. It can be seen that for small and intermediate $\phi$ the theoretical curve with $\lambda=0$ agrees quite well with the experiment, whereas for large $\phi$, the agreement with experiment seems to be better for the theoretical curve with $\lambda=1$. Markedly, the curve with $\lambda=1$ does predict a peak in $\nu_{\mathrm{B}}$ at large $\phi$, though at a slightly higher $\phi$ value and smaller in size than the experiment. The origin of this peak comes from the $\sqrt{\lambda}$ term in equation (2). Obviously the peak will disappear with $\lambda \rightarrow 0$. This comparison shows that the PAA hydrogel system crosses over from no or weak coupling at small and intermediate $\phi$ to maximum coupling at large $\phi$. This is in contrast to the inference from the results of PVA and PVC gels that $\lambda \simeq 0$ throughout the whole range of $\phi$ [1]. Treating $\lambda$ as an adjustable parameter, it is found that in order to describe the experimental data of $\nu_{\mathrm{B}}, \lambda$ has to vary according to figure 5 . This behaviour of $\lambda$ confirms the above observation that the degree of coupling between the elastic waves in the network and fluid changes from weak to strong coupling at high $\phi$, except that it changes back to weak coupling again as $\phi$ approaches 1 .

The prediction of the theory for $\Gamma_{\mathrm{B}}$ is shown in figure 3 as a line using the $\lambda$ values of figure 5 and the physical constants in table I. A constant value for the frictional damping of $f \simeq$ $1.6 \times 10^{13} \mathrm{kgm}^{-3} \mathrm{~s}^{-1}$ has been chosen. The theory successfully predicts the essential qualitative features of $\Gamma_{\mathrm{B}}$, in particular the presence of a maximum, though at a somewhat higher $\phi$ value than the experiment. In the same spirit of the approach taken for $\nu_{\mathrm{B}}$, we may also treat $f$ as an adjustable parameter. A better agreement is obtained if $f$ is to vary with $\phi$ as shown in figure 5. It is to be noted that the behaviours of $\lambda$ and $f$ as a function of $\phi$ are rather similar, both exhibit a maximum at about the same $\phi$ value which coincides with that at which $\nu_{\mathrm{B}}$ is a maximum. We know of no reason why $\lambda$ and $f$ should vary thus, but believe this coincidence is not accidental. We are currently investigating the possibility of studying other systems which may show similar behaviours. However, gelation systems with good optical quality, wide range of $\phi$ and especially with large $\lambda$ are very difficult to find.

In the MD theory, Brillouin light scattering from polymer gels are analysed in terms of a model which treats the motions of the fluid and the network separately and on equal footing. The merit of the dynamical set adopted by Marqusee and Deutch in their hydrodynamic equations for gels is that the essential physics is contained (i) in the simple frictional damping term proportional to $f$ that describes the exchange of momentum between the fluid and the 

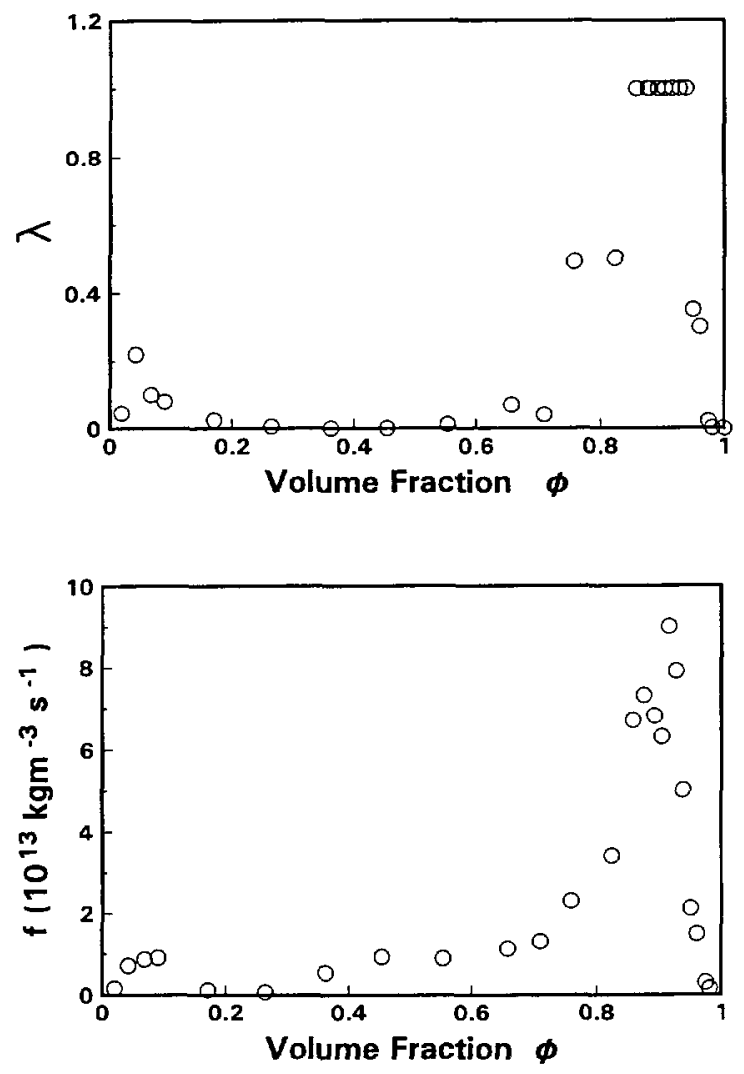

Fig. 5. - The dependence of $\lambda(\phi)$ to reproduce the data of figure 1 and the dependence of $f(\phi)$ to reproduce the data of figure 3 (see text).

network and (ii) the parameter $\lambda$ which describes the coupling of the elastic waves in the fluid and in the network [4]. It is these terms that are carrying information about the polymer network into the position and width of the Brillouin peak. There are other, simpler or more complicated, hydrodynamic descriptions which are consistent with the symmetry and physics of gels. For instance, the hydrodynamic equations introduced by Bacri et al. $[13,14]$ in their study of ultrasonic attenuation in gels do not include the coupling of elastic waves described by $\lambda$. Nevertheless for $\lambda=0, M D$ description reduces to that of Bacri et al. Since all the known similar studies on other gels $[2,3,10]$ do not exhibit any peak in $\nu_{\mathrm{B}}$ at high $\phi$ values, they may be described by the MD theory with $\lambda=0$, as we reported on two of them recently [1]. However they are not good systems to verify fully the MD theory. The present study on PAA hydrogel clearly demonstrates the role played by the coupling parameter $\lambda$ in the behaviour of $\nu_{\mathrm{B}}$. We believe this is the first observation of such an effect. According to MD theory the frictional coefficient $f$ is likely to be a complicated, nonanalytic function of both the volume fraction $\phi$ of the gel network and the frequency of the mechanical disturbance. We have found that not only $f$ but also $\lambda$ are complicated functions of $\phi$. Johnson [15] has applied the Biot theory $[16,17]$ of acoustics in porous media to the gel problem. In the work of Biot 
and that of Johnson, there is additional inertial coupling besides that introduced by Marqusee and Deutch. We have used the Johnson theory to analyse the PVC gel [3]. However, contrary to expectation, it fails to account for the observations in the gelation system. In particular, the tortuosity parameter calculated from the theory is nearly always less than unity which is a physical unreasonable results [3]. This is why no attempt has yet been made to analyse the present results in accordance with the Johnson theory.

\section{Conclusion.}

Our measurements of the dependence of the Brillouin shift $\nu_{B}$ and width $\Gamma_{B}$ on volume fraction $\phi$ for PAA hydrogel have revealed new features which have not been observed in the previous similar studies on other gels $[2,3,10]$. In particular, a prominent peak has been observed in $\nu_{\mathrm{B}}$ at high $\phi$ value. The results have been compared with the theory of Marqusee and Deutch for Brillouin light-scattering from polymer gels, incorporated with a new assumption that the bulk modulus of the gels scales as $\phi$. It is found that the measurements are roughly consistent with the essential features of the predictions of the theory for the case of strong frictional damping $f$. However in order to obtain a realistic description of the experimental data in terms of this theory it is found that both the coupling parameter $\lambda$ and frictional damping $f$ must be forced to depend strongly on $\phi$ instead of assuming constant values as we have done in our previous analysis of PVA and PVC gels [1]. In fact, both $\lambda$ and $f$ exhibit a maximum at about the same the $\phi$ value where $\nu_{B}$ is also maximum. It is hoped that the data obtained for $\lambda$ and $f$ in this paper will stimulate further theoretical investigation into the behaviour of these parameters as functions of $\phi$ in polymer gels.

\section{References}

[1] Ng S.C., Li Y., J. Phys II France 3 (1993) 1241.

[2] Ng S.C., Hosea T.J.C., Gan L.M., J. Phys. France Lett. 46 (1985) L-887.

[3] Ng S.C., Hosea T.J.C., Teoh S.H., Chem. Phys. 110 (1986) 225.

[4] Marqusee J.A., Deutch J.M., J. Chem. Phys. 75 (1981) 5239.

[5] Michaeli I., Katchalsky A., J. Polym. Sci. 23 (1957) 683.

[6] Harada J., Chern R.T., Stannett V.T., Polyelectolyte gels; R.S. Harland, R.K. Prud'homme Eds. (ACS symposium series, 1992) p. 80.

[7] Molyneux P., Water-soluble Synthetic Polymers: Properties and Behaviour, Vol I (CRC press Inc., 1983) p. 75.

[8] Ng S.C., Hosea T.J.C., Teh H.C., Gan L.M., J. Phys. E: Sci Instrum. 18 (1985) 250.

[9] Hosea T.J.C., Ng S.C., Oates C.G., Food Hydrocolloids 4 (1990) 137.

[10] Brown W., Schillen K., Johnsen R., Konak C., Dvoranek L., Macromolecules 25 (1992) 802.

[11] Brandrup J., Immergut E.H., Polymer Handbook (Wiley, 1989).

[12] de Gennes P.G., Scaling Concepts in Polymer Physics (Cornell University Press, 1979).

[13] Bacri J.C., Rajaonarison R., J. Phys. France Lett. 40 (1978) 5.

[14] Bacri J.C., Courdille J.M., Dunnas J., Rajaonarison R., J. Phys. France Lett. 41 (1980) 372.

[15] Johnson D.L., J. Chem. Phys. 77 (1982) 1531.

[16] Boit M.A., J. Acount. Soc. Am. 28 (1956) 168.

[17] Boit M.A., J. Appl. Phys. 33 (1962) 1482.

[18] Dorsey N.E., Properties of Ordinary Water-Substance (Reinhold Publishing Corporation, 1940).

[19] Rouch J., Lai C.C., Chen S.H., J. Chem. Phys. 66 (1977) 5031. 